\title{
An investigation into the performance of four cloud droplet activation parameterisations
}

\author{
E. Simpson, P. Connolly, and G. McFiggans \\ Centre for Atmospheric Science, School of Earth, Atmospheric and Environmental Sciences, University of Manchester, \\ Manchester, M13 9PL, UK \\ Correspondence to: G. McFiggans (g.mcfiggans@manchester.ac.uk)
}

Received: 16 January 2014 - Published in Geosci. Model Dev. Discuss.: 10 February 2014

Revised: 9 June 2014 - Accepted: 13 June 2014 - Published: 24 July 2014

\begin{abstract}
Cloud droplet number concentration prediction is central to large-scale weather and climate modelling. The benchmark cloud parcel model calculation of aerosol particle growth and activation, by diffusion of vapour to aerosol particles in a rising parcel of air experiencing adiabatic expansion, is too computationally expensive for use in largescale global models. Therefore the process of activation of aerosol particles into cloud droplets is parameterised with an aim to strike the optimum balance between numerical expense and accuracy. We present a detailed systematic evaluation of three cloud droplet activation parameterisations that are widely used in large-scale models and one recent update. In all cases, it is found that there is a tendency to overestimate the fraction of activated aerosol particles when the aerosol particle "median diameter" is large (between 250 and $2000 \mathrm{~nm}$ ) in a single lognormal mode simulation. This is due to an infinite "effective simulation time" of the parameterisations compared to a prescribed simulation time in the parcel model. This problem arises in the parameterisations because it is assumed that a parcel of air rises to the altitude where maximum supersaturation occurs, regardless of whether this altitude is above the cloud top. Such behaviour is problematic because, in some cases, large aerosol can completely suppress the activation of drops. In some cases when the "median diameter" is small (between 5 and $250 \mathrm{~nm}$ ) in a single lognormal mode the fraction of activated drops is underestimated by the parameterisations. Secondly, it is found that in dual-mode cases there is a systematic tendency towards underestimation of the fraction of activated drops, which is due to the methods used by the parameterisations to approximate the sink of water vapour.
\end{abstract}

\section{Introduction}

Clouds are important components in understanding climate change and therefore must be accurately represented in largescale (regional and global) weather and climate models so that we can make realistic future climate predictions. The effective radiative forcing of aerosol and cloud interactions (including cloud albedo enhancement and cloud lifetime effect) have some of the largest uncertainties of all considered components of radiative forcing as reported in the IPCC 5th Annual Report (Myhre et al., 2013, p. 123, Fig. 8.20). Aerosol particles interact with clouds by acting as nuclei on which water vapour can condense under liquid water supersaturated conditions. A change in the concentration of the subset of aerosol particles that act as cloud condensation nuclei (CCN) within a cloud will lead to a change in cloud droplet number concentration. The albedo of a cloud is dependent on the number concentration of cloud drops (Twomey, 1974) as is the cloud lifetime (Albrecht, 1989). It is therefore key to understanding the role of clouds in climate that the activation of cloud droplets is well represented in numerical models.

The ability of a particle to act as a CCN is dependent on its size, composition and the ambient conditions - most notably the supersaturation (Pruppacher and Klett, 1997). Köhler theory determines the size to which an involatile deliquesced particle must grow in order to activate as a cloud drop, which is determined by the particle dry size, composition and the ambient supersaturation.

The presence of large (typically larger than $1000 \mathrm{~nm}$ dry size) aerosol particles may suppress the number concentration of activated drops. This is due to the fact that larger drops compete effectively for available water vapour such that they suppress the maximum supersaturation $\left(S_{\max }\right)$, which results 
in fewer "smaller" particles activating (e.g. Ghan et al., 1998; Sander, 1999). Some large particles do not grow quickly enough to reach their critical diameter before peak supersaturation and therefore remain as unactivated particles, reducing the total fraction of activated drops. The large amount of water condensing in the growth of large particles leads to a suppression of $S_{\max }$.

Sectional cloud parcel models provide a physically realistic and internally consistent calculation of particle activation and droplet growth in a parcel of air undergoing adiabatic ascent. In this study we use the Aerosol-Cloud-Precipitation Interaction Model (ACPIM) (Connolly et al., 2012). ACPIM calculates both the sub-saturated growth of aerosol particles as well as their supersaturated growth by water vapour diffusion. This model makes very few simplifications of the condensation process giving confidence that the predictions are physically realistic. We acknowledge that the dynamical framework employed in this work does not allow reproduction of realistic atmospheric dynamics, nevertheless the initial formation period of clouds can often be assumed to be adiabatic (Heymsfield et al., 1978). For a detailed description of processes represented in ACPIM, that are relevant to this paper, see the supplementary information of Topping et al. (2013).

Cloud parcel models such as ACPIM are too computationally expensive to be used in large-scale global climate models. It is therefore necessary to rely on parameterisation schemes to estimate the number of activated cloud drops within large-scale models. The most widely used parameterisation schemes fall into two families - those based on the work of Abdul-Razzak et al. (1998), Abdul-Razzak and Ghan (2000) and those following Fountoukis and Nenes (2005). The differences between these two sets of parameterisations are discussed in Sect. 2.1. A synopsis of these parameterisations is given in Connolly et al. (2013).

Ghan et al. (2011) provide an evaluation of two of the parameterisations evaluated in this study: Abdul-Razzak and Ghan (2000) and Fountoukis and Nenes (2005) with Barahona et al. (2010) extension. In this work we explore the performance of the parameterisations over a larger parameter space and run many more simulations. In general our results are similar to those of Ghan et al. (2011): AbdulRazzak and Ghan (2000) consistently underestimate the fraction of activated drops in a dual-mode aerosol size distribution and Fountoukis and Nenes (2005), with Barahona et al. (2010) extension, underestimates the fraction of activated drops to a lesser extent than Abdul-Razzak and Ghan (2000), in simulations where the total number concentration of aerosol in a dual-mode size distribution is $>2000 \mathrm{~cm}^{-3}$. Ghan et al. (2011) make a suggestion for further work to provide a comparison of parameterisations against different numerical models such as the results presented here.
Table 1. Initial conditions for the simulations.

\begin{tabular}{lccc}
\hline Temperature & Pressure & Runtime (ACPIM only) & RH \\
\hline $290.15 \mathrm{~K}$ & $950 \mathrm{hPa}$ & $2000 \mathrm{~s}$ & 0.90 \\
\hline
\end{tabular}

\section{Method}

ACPIM is a detailed bin-resolving cloud parcel model and is taken as the benchmark for comparison of the parameterisation methods. It is therefore used as the reference model for this study. The three widely used parameterisation schemes and one recent update are evaluated for their ability to reproduce accurate values of the fraction of activated drops, for a large parameter space. We now explain the salient points of the parameterisations.

\subsection{Description of parameterisations}

Each of the parameterisation schemes used in this study can be used to represent the activation of single or multiple lognormal aerosol size distributions or "modes". A lognormal distribution, describing the number of aerosol particles per natural logarithm of the bin width, $\frac{\mathrm{d} N}{\mathrm{~d} \ln D_{p}}$, is described by the following equation:

$\frac{\mathrm{d} N}{\mathrm{~d} \ln D_{p}}=\frac{N_{\mathrm{ap}}}{\ln \sigma \sqrt{2 \pi}} \exp \left[-\frac{\ln ^{2}\left(\frac{D_{p}}{d_{\mathrm{m}}}\right)}{2 \ln \sigma^{2}}\right]$,

where $N_{\text {ap }}$ is the total number concentration of aerosol particles, $\ln \sigma$ is the natural logarithm of the geometric standard deviation and $d_{\mathrm{m}}$ is the median diameter (Jacobson, 1999). The values for the median aerosol diameter of a lognormal mode, $d_{\mathrm{m}}$, are given in Table 2 along with the breadth of the mode, $\ln \sigma$.

The first scheme, originally described in Abdul-Razzak et al. (1998), is further developed in Abdul-Razzak and Ghan (2000) to include multiple modes, hereafter referred to as ARG. The second scheme is of Fountoukis and Nenes (2005), hereafter referred to as FN, and the third is an extension of FN that includes the effects of large (giant) $\mathrm{CCN}$ described in Barahona et al. (2010), hereafter referred to as FN GCCN. The fourth scheme is an update to the FN and FN GCCN schemes by Morales Betancourt and Nenes (2014), hereafter referred to as FN GCCN BM.

The ARG and FN families of parameterisations find approximate values for the maximum supersaturation achieved by a rising parcel of air, $S_{\max }$, in different ways. FN sets the equation for the rate of change of supersaturation to zero and then iteratively finds a value for $S_{\max }$ that satisfies the equation. This is done by using a method called "population splitting" to divide the size distribution of $\mathrm{CCN}$ into two groups: one with only CCN that are close to their critical diameter and the other with $\mathrm{CCN}$ that are not (Fountoukis and Nenes, 
2005). ARG also sets the equation for the rate of change of supersaturation to zero, then after neglecting the effects of curvature, gas kinetics and solute (in the equation for droplet radius growth rate) an approximate expression for $S_{\max }$ is derived (Abdul-Razzak et al., 1998). ARG is written in terms of dimensionless parameters to account for the errors made by simplifying the droplet growth rate (see Abdul-Razzak et al., 1998, for details).

ARG approximates the maximum supersaturation by assuming that all particles start at their equilibrium size and then grow further depending on the supersaturation. FN splits the population of aerosol into two separate groups: those particles that are small and are therefore assumed to start at their equilibrium size and then grow further depending on the supersaturation, (similar to ARG) and those particles that are large and take time to grow to their equilibrium size before growing further. This method takes into account kinetic limitations to the growth of larger particles.

In both parameterisations the number of $\mathrm{CCN}$ that activate given the maximum supersaturation is then calculated by applying Köhler theory to make a change of variable of $S_{\max }$ to $D_{p}$, which is then used in conjunction with the prescribed lognormal size distributions to calculate the number of activated particles. The number of activated aerosol determined by both types of schemes, ARG and FN, is considered to be the number of aerosol with diameter greater than the smallest activated aerosol diameter (Abdul-Razzak et al., 1998; Fountoukis and Nenes, 2005).

Barahona et al. (2010), further develop the FN method to account for the fact that giant $\mathrm{CCN}-$ i.e. $\mathrm{CCN}$ with dry aerosol diameters greater than approximately $500 \mathrm{~nm}$ - may have insufficient time to grow to their activation size.

A recent update to the $\mathrm{FN}$ parameterisation and the Barahona et al. (2010) development has been made by Morales Betancourt and Nenes (2014). This update aims to better account for the growth of inertially limited particles and their subsequent contribution to the water vapour sink, by only allowing the growth of the largest particles to be calculated by the Barahona et al. (2010) parameterisation (Morales Betancourt and Nenes, 2014). The update also adds an additional term to the equation for the rate of change of supersaturation that allows for a smoother transition between the two populations of aerosol created by the population splitting technique (Morales Betancourt and Nenes, 2014).

\subsection{Model inputs}

ACPIM allows the size distribution and any variation of composition of aerosol to be defined in addition to the particle mixing state. Updraft velocity is prescribed and the rate of change of pressure with respect to time, $\frac{\mathrm{d} P}{\mathrm{~d} t}=-\frac{P}{T R_{\mathrm{a}}} g w$, is determined assuming an atmosphere in hydrostatic balance. Importantly, ACPIM is time dependent and the maximum simulated ascent time in the model is controlled. This ensures that the height that the parcel rises and hence the cloud depth for the simulation (for a given updraft velocity) is held within atmospherically reasonable bounds. The model assumes moist adiabatic ascent, no mixing with ambient air and outputs the supersaturation and the number distribution of activated and unactivated aerosol particles as a function of time. Only aerosol with diameters larger than their critical diameter are recorded as activated drops. Here we use the number of activated drops at the end of the simulation time in the parcel model to compare with the number of activated drops calculated by the parameterisations.

Evaluations of all of the mentioned parameterisation schemes against detailed numerical parcel models are reported in their respective studies. In this study we use a similar evaluation method to Barahona et al. (2010). However, to avoid any bias in our results we use a Monte Carlo sampling technique to explore the parameter space over an atmospherically relevant range of conditions.

Barahona et al. (2010) use a very wide parameter space, where the number concentration of the two lognormal modes and the median aerosol diameter in the first mode are chosen from Seinfeld and Pandis (1998) to be atmospherically representative (p. 2470, Barahona et al., 2010). The range of median aerosol diameters investigated in their study is chosen to represent all possible sizes of aerosol, from that of newly nucleated particles to giant CCN (as described by Pruppacher and Klett, 1997).

Here we have chosen a large parameter space for the single-mode case similar to the one used by Barahona et al. (2010) to enable us to demonstrate the accuracy of the parameterisations under many conditions.

In the dual-mode case the parameter space is also similar to that used by Barahona et al. (2010). In the Supplement of this paper the parameter space used in dual-mode experiments has been reduced to avoid extreme concentrations of small and large particles (that are rarely found in the atmosphere) and a smaller range of updraft velocities so that experiments only represent cloud depths more reasonably likely to exist. Such parameter space reduction reduces potential biases in the parameterisations that would be driven by unphysical parameter combinations.

The values in Table 1 are similar to the conditions used in the evaluations of ARG, FN and FN GCCN in their respective studies. Similarly, the values in Table 2 were chosen to be within the same parameter space as was used to initially evaluate ARG, FN and FN GCCN. A value of 1 is used for the mass accommodation coefficient of water in accordance with the latest experimental evidence (Miles et al., 2012). 
Table 2. Parameter space investigated for both ACPIM and the parameterisations. Two evaluations were done: single mode and dual mode experiments.

\begin{tabular}{llll}
\hline & & \multicolumn{2}{c}{ Dual-mode experiment } \\
\cline { 3 - 4 } Variable & Single-mode experiment & MODE 1 & MODE 2 \\
\hline Number conc. of aerosol & $50-2000 \mathrm{~cm}^{-3}$ & $2000 \mathrm{~cm}^{-3}$ & $400 \mathrm{~cm}^{-3}$ \\
$\ln \sigma$ & $0.2-0.8$ & 0.46 & 0.46 \\
Median diameter & $50-1000 \mathrm{~nm}$ & $80 \mathrm{~nm}$ & $5-5000 \mathrm{~nm}$ \\
Updraft velocity & $0.01-10 \mathrm{~m} \mathrm{~s}^{-1}$ & $0.01-10 \mathrm{~m} \mathrm{~s}^{-1}$ & $0.01-10 \mathrm{~m} \mathrm{~s}^{-1}$ \\
\hline
\end{tabular}

\section{Results}

\subsection{Demonstration of time dependency in model simulations}

First, to explore and illustrate the time dependency in the growth of larger aerosol, ACPIM was run for two cases: small aerosol median diameter $(100 \mathrm{~nm}$, the "small aerosol" case) and large aerosol median diameter $(1000 \mathrm{~nm}$, the "large aerosol" case), both with total number concentrations of $500 \mathrm{~cm}^{-3}$. The results from the parameterisations and ACPIM are shown in Fig. 1, for the same initial conditions.

In the "small aerosol" case the parameterisations reproduce the fraction of activated drops and maximum supersaturation well. However, in the "large aerosol" case, the parcel model does not reach the maximum possible supersaturation because it takes too long for the larger aerosol to reach the size required for activation. The result is that no aerosol activate in the simulated time of $2000 \mathrm{~s}$ for the large aerosol case. The results from the parameterisations effectively have no run time limit and therefore activate nearly all of the large aerosol. This would be equivalent to running ACPIM for an unrealistically long time such that the parcel of air reaches an unrealistic height before activating the large particles into cloud drops. We refer to this as an "infinite effective simulation time" artifact for the case of the parameterisations. Figure S1 in the Supplement shows that the actual amount of time the parcel model requires to activate the same fraction of large drops as the parameterisations, $\sim 0.8$, is $\sim 56000 \mathrm{~s}$. This equates to $16.8 \mathrm{~km}$ for the updraft velocity of $0.3 \mathrm{~m} \mathrm{~s}^{-1}$ that was used. Unperturbed ascent of this extent does not happen in the atmosphere; hence, the parameterisation appears to activate an unphysical fraction of the particles under these conditions (albeit with a population of unphysically large particles). Elimination of unrealistically high number concentrations of large particles from our simulations ensures that such obvious biases are not introduced in our evaluation, but the effect of overestimated activated fractions with unrealistic "effective simulated time" will still occur. This is a feature throughout the comparisons presented in the following sections.
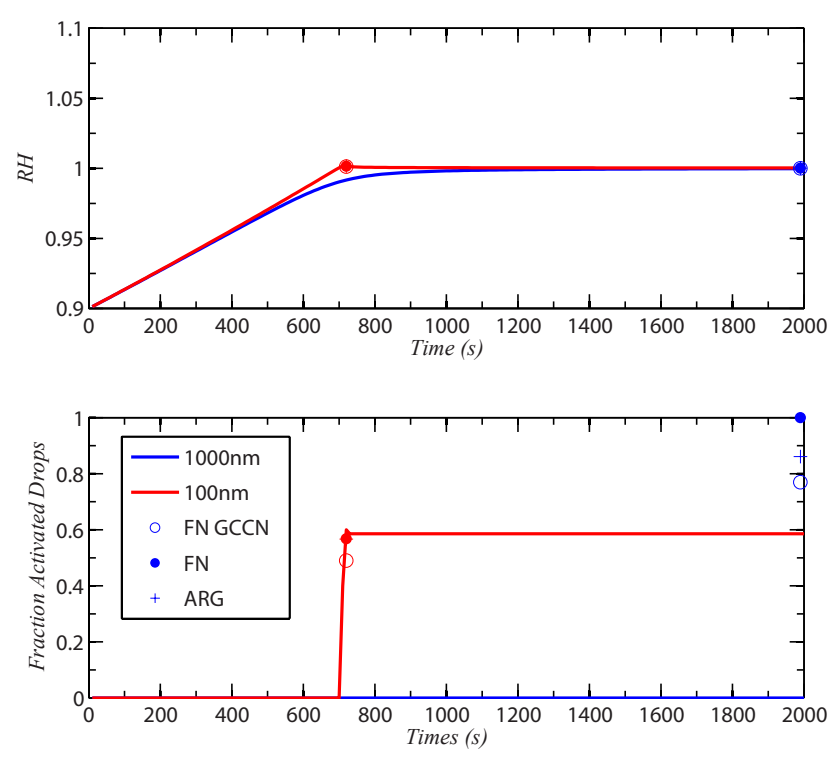

Figure 1. Time series of RH (top panel) and fraction of activated drops (bottom panel) as calculated by ACPIM with initial conditions described in Table 1 , a number concentration of aerosol $500 \mathrm{~cm}^{-3}$, with median aerosol diameters $100 \mathrm{~nm}$ (red) and $1000 \mathrm{~nm}$ (blue). Using the same initial conditions, results from the parameterisations are plotted as single points at the time of maximum supersaturation (which is calculated from the parcel model).

\subsection{Single-mode experiment}

1500 simulations were conducted using single lognormal modes of particles randomly selected from the parameter ranges shown in Table 2.

Figure 2 shows that for aerosol median diameters, $d_{\mathrm{m}}$, of less than $\sim 300 \mathrm{~nm}$ the fraction of activated drops calculated by the parameterisations agrees reasonably well with the parcel model - see also Fig. S2 in the Supplement. Figure $\mathrm{S} 2$ shows results from simulations with high concentrations $\left(10000 \mathrm{~cm}^{-3}\right)$ of aerosol with small median diameters $(5-250 \mathrm{~nm})$ and that there is a general tendency to underestimate the fraction of activated drops at small sizes. At larger sizes $\left(d_{\mathrm{m}}>300 \mathrm{~nm}\right)$ the parameterisations tend to overestimate the fraction of activated drops - see also Fig. S3 in the Supplement which shows results from simulations with low 


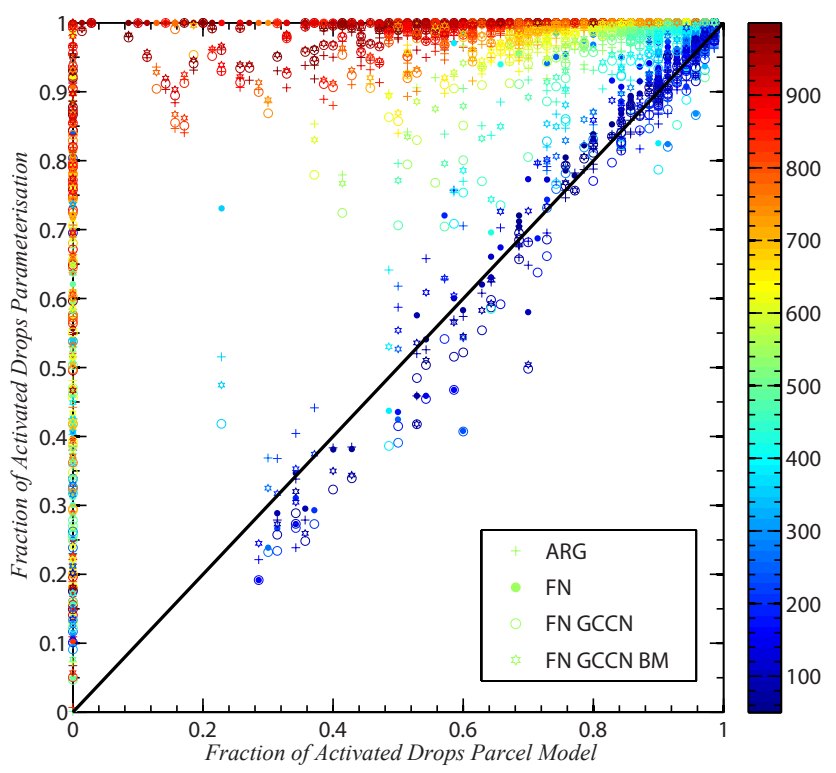

Figure 2. Results from 1500 runs with 1 lognormal mode of ammonium sulfate aerosol with randomly sampled variable values as detailed in Table 2 and initial conditions described in Table 1. Symbols are coloured by median aerosol diameter ( $\mathrm{nm})$.

concentrations $\left(100 \mathrm{~cm}^{-3}\right)$ of aerosol with large median diameters $(250-2000 \mathrm{~nm})$. This overestimation of the parameterisation relative to the parcel model increases as the median diameter increases for values of $d_{\mathrm{m}}>300 \mathrm{~nm}$. In many cases the parameterisations activate all of the available aerosol where the parcel model only activates a small fraction of available aerosol. In other cases the parcel model does not activate any aerosol where the parameterisations do. This is due to the unrealistic "effective simulation time" effect described in Sect. 3.1.

No clear relationship between $\ln \sigma$, the total number concentration of aerosol, or the fraction of activated drops can be drawn from the results of this study - see also Figs. S4 and S5 in the Supplement which are similar to Fig. 2 with data points colour coded by $\ln \sigma$ and aerosol number concentration respectively.

\subsection{Dual-mode experiment}

Using a similar parameter space as that used in Barahona et al. (2010) a further 1500 simulations were conducted with two lognormal modes of aerosol. Figure 3 has a very noticeable feature: the overestimation of the fraction of activated aerosol by all three parameterisations below approximately 0.16 fraction activated. The value of 0.16 is equal to the fraction of the total aerosol loading comprising the second aerosol mode $\left(400 \mathrm{~cm}^{-3}: 2400 \mathrm{~cm}^{-3}\right)$. In many cases (mostly at updraft velocities above $2 \mathrm{~m} \mathrm{~s}^{-1}$ ) the parameterisations activate all of the second mode and none of the first mode. At updraft velocities between 2 and $6 \mathrm{~m} \mathrm{~s}^{-1}$ this

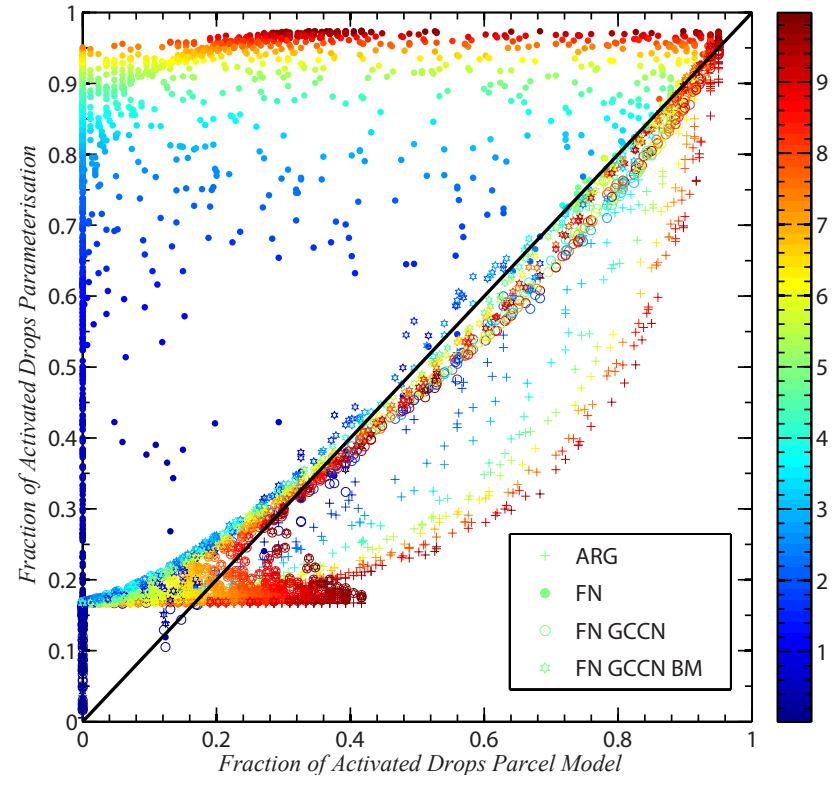

Figure 3. Results from 1500 runs with a bimodal aerosol size distribution. Only the median diameter of aerosol in the second mode and updraft velocity were changed between runs. Symbols are coloured by updraft velocity, $\mathrm{m} \mathrm{s}^{-1}$, see colour bar. For initial conditions and parameter ranges see Tables 1 and 2 respectively.

results in an overestimation of the fraction of activated drops and at updraft velocities larger than $6 \mathrm{~m} \mathrm{~s}^{-1}$ an underestimation. This is a feature of the time-independent nature of the parameterisations, which can be demonstrated by increasing the run time of the parcel model from 2000 to $8000 \mathrm{~s}$ - see Fig. 4, which shows that the feature at 0.16 fraction activated, present in Fig. 3, is less pronounced with a longer run time. It should be noted that we had to reduce the maximum values of $w, d_{\mathrm{m}}$ of the second mode and number concentration in the second mode. For the larger values realistic cloud base altitudes were not modeled and hence should not be compared to parameterisations.

In cases where the fraction of activated drops is greater than 0.16 in the parcel model FN GCCN performs well with a generally small underestimation. FN GCCN BM also preforms well with generally less of an underestimation than FN GCCN and slight overestimation at low updraft velocities, $w \lesssim 4 \mathrm{~m} \mathrm{~s}^{-1}$. ARG underestimates the fraction of activated drops significantly more than FN GCCN and the underestimation increases with updraft velocity: there is implicitly too much competition for water vapour in its formulation, which arises from the assumption that all particles start at their equilibrium size. This is the opposite effect to that exhibited by FN, implying too little competition in this scheme. As mentioned previously, the FN scheme uses "population splitting" to divide particles into two groups: those that are free from kinetic limitations to growth and those where kinetic limitations dominate. $S_{\text {part }}$ is estimated as the division in an aerosol 


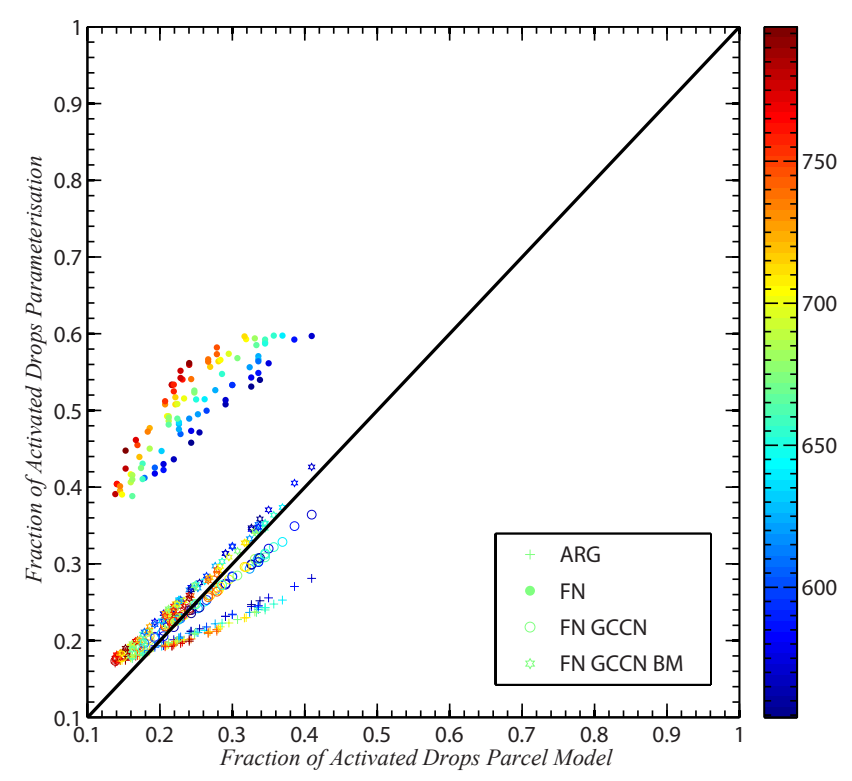

Figure 4. Results from 100 runs with bimodal aerosol size distributions, $500 \mathrm{~cm}^{-3}$ in the first mode and $100 \mathrm{~cm}^{-3}$ in the second and a run time of $8000 \mathrm{~s}$. Only the median diameter of the aerosol in the second mode and the updraft velocity were changed between runs, within the ranges of $550 \leq d_{\mathrm{m}} \leq 800 \mathrm{~nm}$ and $0.2 \leq w \leq 0.5 \mathrm{~m} \mathrm{~s}^{-1}$ respectively. Symbols are coloured by median diameter $(\mathrm{nm})$ of the second mode, see colour bar. For initial conditions see Table 1.

population between these two groups and is estimated using an empirical function derived from parcel model simulations over a limited range of aerosol characteristics. It would appear that this function is not appropriate for all aerosol distributions tested here and results in two little competition for water vapour in this scheme. The method for approximating the integral for $S_{\max }$ in ARG is therefore too negative and in FN, too positive. FN GCCN corrects this with an additional term in the integral (Barahona et al., 2010).

As expected, ARG also underestimates the peak in RH in the majority of cases (see Fig. 5 that shows results of peak $\mathrm{RH}$ achieved in each simulation in the dual-mode case with a limited parameter space), which also shows that FN GCCN and FN GCCN BM perform best out of the three parameterisations at predicting the peak RH. Figure 5 also shows that the spread of peak RH values calculated by the parameterisations increases with updraft velocity, rather than a strong systematic offset.

The difference between FN GCCN and FN can clearly be seen in Fig. 3. Without the inclusion of effects of large aerosol in the parameterisation, FN overestimates the number of activated drops and this overestimation increases with median diameter of second aerosol mode (see Fig. S6 in the Supplement) and updraft velocity. Since the FN GCCN scheme shows a marked improvement when compared with FN in cases where large aerosol particles are present we have excluded the FN results in the comparison between the single

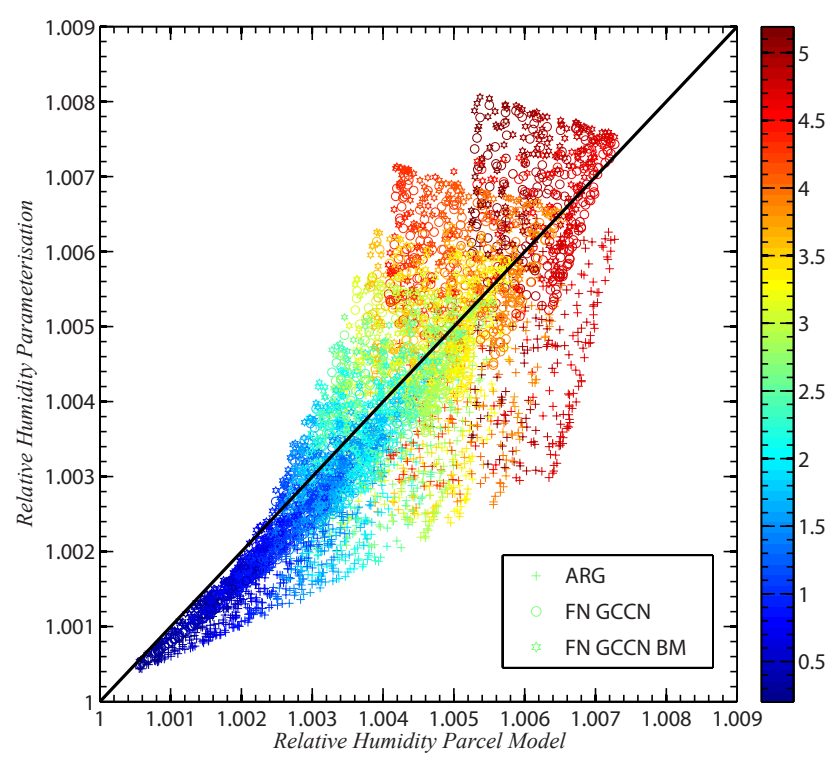

Figure 5. Results for peak RH from 1500 runs with bimodal aerosol size distributions. Only the median diameter of the aerosol in the second mode and updraft velocity were changed between runs within the ranges of $100 \leq d_{\mathrm{m}} \leq 800 \mathrm{~nm}$ and $0.2 \leq w \leq 5 \mathrm{~m} \mathrm{~s}^{-1}$ respectively. Symbols are coloured by updraft velocity, see colour bar. For initial conditions see Table 1.

and dual mode experiments below. Also as FN GCCN and FN GCCN BM are very similar only one, FN GCCN, is used in the comparison below.

\subsection{Comparison between single- and dual-mode cases}

Figure 6 shows that the ratio of the number of activated drops calculated by the model to the number calculated by the parameterisations is most often close to unity for the monomodal cases. The majority of the rest of the data for the monomodal case are found below 1 showing a general overestimation by the parameterisations of the fraction of activated drops. All data in the first bin show where the parcel model does not activate any drops but the parameterisations do. This overestimation occurs because the growth of relatively large particles, $d_{\mathrm{m}}>250 \mathrm{~nm}$, should be inertially limited. Although correcting for this in bimodal cases, FN GCCN does not preform well in monomodal cases. The bimodal case shows a tendency to underestimate the number of activated drops using both ARG and FN GCCN, but with ARG clearly performing less well. In this case a significant fraction of particles are very large and therefore far from their activation size. ARG overestimates the vapour sink by assuming that all particles start at their equilibrium size, resulting in an underestimation of the number of activated drops. Here FN GCCN effectively corrects for the inertially limited growth of large particles and therefore does not overestimate the vapour sink as ARG does. 


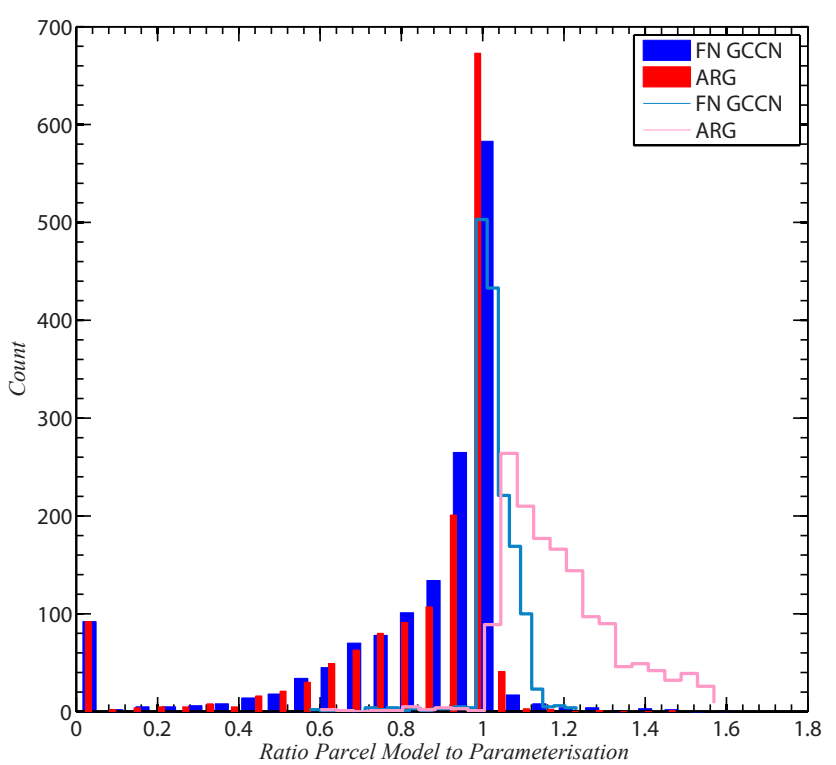

Figure 6. Histogram of the ratio of number of activated drops calculated by the parcel model to the number of activated drops calculated by FN GCCN and ARG parameterisations. Solid bars for monomodal runs and lines for bimodal runs.

\section{Conclusions}

While the parameterisations evaluated in this paper perform well under a range of atmospherically relevant conditions, they also produce results that differ notably from the results of the parcel model under a wide range of conditions. Such conditions could provide the input distributions for the parameterisations when used within global climate models, producing significantly unphysical estimates of activated drop number concentrations.

The main conclusions from this study are as follows.

- First, there is a systematic tendency in the parameterisations towards overestimating the activated fraction of drops when the median aerosol particle diameter is large, $\gtrsim 250 \mathrm{~nm}$. This is a result of parcel models considering the time required for activation and cloud development, whilst the parameterisations implicitly allow an infinite "effective simulation time".

- Second, the estimation of $S_{\max }$ within the parameterisations leads to apparent opposite behaviour between the ARG and FN families of parameterisations. FN GCCN performs better with the additional term in the $S_{\max }$ integral approximation. There is a small residual tendency towards underestimation of the fraction of activated drops, but with less of a low bias than ARG. FN GCCN BM accounts for this slight low bias by only including the largest particles in the Barahona et al. (2010) development, however this does lead to a slight overestimation in the fraction of activated drops in some low-updraft cases. The ARG parameterisation represents too much competition for water vapour resulting in an underestimation of the fraction of activated drops.

Hence, in a general sense, the parameterisations evaluated here tend to overestimate the number of activated drops in a single lognormal aerosol size distribution and underestimate the number of activated drops in dual lognormal aerosol size distribution. The overestimation in the single-mode case is a result of an infinite "effective simulation time" in the parameterisations. The underestimation in the dual-mode case results from the methods used to approximate $S_{\max }$ within the parameterisations.

Due to the substantial improvement that the Barahona et al. (2010) amendment makes to the FN parameterisation, we recommend that the effects of large unactivated particles on the maximum supersaturation be included in cloud models.

It should be noted that the performance of the parameterisations is very dependent on the parameter ranges chosen for the comparisons as illustrated throughout the Supplement for both single- and dual-mode simulations.

\section{The Supplement related to this article is available online at doi:10.5194/gmd-7-1535-2014-supplement.}

Acknowledgements. This work was funded by the NERC ACIDPRUF programme, grant code NE/I020121/1, NCAS and a NERC studentship.

Edited by: H. Tost

\section{References}

Abdul-Razzak, H. and Ghan, S. J.: A Parameterization of Aerosol Activation, Part II: Multiple Aerosol Types, J. Geophys. Res. 105, 6837-6844, doi:10.1029/1999JD901161, 2000.

Abdul-Razzak, H., Ghan, S. J., and Rivera-Carpio, C.: A parameterization of aerosol activation. 1. Single aerosol type, J. Geophys. Res., 103, 6123-6131, 1998.

Albrecht, B. A.: Aerosols, cloud microphysics and fractional cloudiness, Science, 245, 1227-1230, 1989.

Barahona, D., West, R. E. L., Stier, P., Romakkaniemi, S., Kokkola, H., and Nenes, A.: Comprehensively accounting for the effect of giant $\mathrm{CCN}$ in cloud activation parameterizations, Atmos. Chem. Phys., 10, 2467-2473, doi:10.5194/acp-10-24672010, 2010.

Connolly, P. J., Emersic, C., and Field, P. R.: A laboratory investigation into the aggregation efficiency of small ice crystals, Atmos. Chem. Phys., 12, 2055-2076, doi:10.5194/acp-12-20552012, 2012.

Connolly, P. J., Topping, D. O., Malavelle, F., and McFiggans, G.: A parameterisation for the activation of cloud drops including the 
effects of semi-volatile organics, Atmos. Chem. Phys. Discuss., 13, 14447-14475, doi:10.5194/acpd-13-14447-2013, 2013.

Fountoukis, C. and Nenes, A.: Continued development of a cloud droplet formation parameterization for global climate models, J. Geophys. Res., 110, doi:10.1029/2004JD005591, erratum, 2005.

Ghan, S. J., Guzman, G., and Abdul-Razzak, H.: Competition between sea salt particles as cloud condensation nulcei, J. Atmos. Sci., 55, 3340-3347, 1998.

Ghan, S. J., Abdul-Razzak, H., Nenes, A., Ming, Y., Xiaohong, L., Ovchinnikov, M., Shipway, B., Meskhidze, N., Xu, J., and Shi, X.: Droplet nucleation: physically-based parameterizations and comparative evaluation, J. Adv. Model. Earth Syst., 3, M10001, doi:10.1029/2011MS000074, 2011.

Heymsfield, A. J., Johnson, P. N., and Dye, J. E.: Observations of moist adiabatic ascent in Northeast Colorado cumulus congestus clouds, J. Atmos. Sci., 35, 1689-1703, 1978.

Jacobson, M. Z.: Fundamentals of Atmospheric Modelling, Cambridge University Press, 1999.

Miles, R. E., Reid, J. P., and Riipinen, I.: Comparison of approaches for measuring the mass accommodation coefficient for the condensation of water and sensitivities to uncertainties in thermophysical properties, J. Phys. Chem. A, 116, 10810-10825, 2012.

Morales Betancourt, R. and Nenes, A.: Droplet activation parameterization: the population splitting concept revisited, Geosci. Model Dev. Discuss., 7, 2903-2932, doi:10.5194/gmdd-7-29032014, 2014.
Myhre, G., Shindell, D., Bréon, F.-M., Collins, W., Fuglestvedt, J., Huang, J., Koch, D., Lamarque, J.-F., Lee, D., Mendoza, B., Nakajima, T., Robock, A., Stephens, G., Takemura, T., and Zhang, H.: Anthropogenic and Natural Radiative Forcing. In: Climate Change 2013: The Physical Science Basis. Contribution of Working Group I to the Fifth Assessment Report of the Intergovernmental Panel on Climate Change, edited by: Stocker, T. F., Qin, D., Plattner, G.-K., Tignor, M., Allen, S. K., Boschung, J., Nauels, A., Xia, Y., Bex, V., and Midgley, P. M., Cambridge University Press, Cambridge, United Kingdom and New York, NY, USA, 2013.

Pruppacher, H. R. and Klett, J. D.: Microphysics of Clouds and Precipitation, 2nd Edn., Kluwer Academic Publishers, Boston, 1997.

Sander, R.: Modeling atmospheric chemistry: interactions between gas-phase species and liquid cloud/aerosol particles., Surv. Geophys., 20, 1-31, 1999.

Seinfeld, J. H. and Pandis, S. N.: Atmospheric Chemistry and Physics, John Wiley and Sons, New York, 1998.

Topping, D., Connolly, P., and McFiggans, G.: Cloud droplet number enhanced by co-condensation of organic vapours, Nat. Geosci., 6, 443-446, doi:10.1038/NGEO1809, 2013.

Twomey, S.: Pollution and the planetary albedo, Atmos. Environ., 8, 1251-1256, 1974. 